\title{
Scaling of peak flows with constant flow velocity in random self-similar networks
}

\author{
R. Mantilla ${ }^{1}$, V. K. Gupta ${ }^{2}$, and B. M. Troutman ${ }^{3}$ \\ ${ }^{1}$ IIHR-Hydroscience \& Engineering, The University of Iowa, Iowa City, IA, 52242, USA \\ ${ }^{2}$ Department of Civil, Environmental and Architectural Engineering, Cooperative Institute for Research in Environmental \\ Sciences, University of Colorado, Boulder, CO, 80309, USA \\ ${ }^{3}$ US Geological Survey, Denver Federal Center, Lakewood, CO, 80225, USA
}

Received: 18 January 2010 - Revised: 15 October 2010 - Accepted: 17 June 2011 - Published: 22 July 2011

\begin{abstract}
A methodology is presented to understand the role of the statistical self-similar topology of real river networks on scaling, or power law, in peak flows for rainfall-runoff events. We created Monte Carlo generated sets of ensembles of 1000 random self-similar networks (RSNs) with geometrically distributed interior and exterior generators having parameters $p_{\mathrm{i}}$ and $p_{\mathrm{e}}$, respectively. The parameter values were chosen to replicate the observed topology of real river networks. We calculated flow hydrographs in each of these networks by numerically solving the link-based mass and momentum conservation equation under the assumption of constant flow velocity. From these simulated RSNs and hydrographs, the scaling exponents $\beta$ and $\phi$ characterizing power laws with respect to drainage area, and corresponding to the width functions and flow hydrographs respectively, were estimated. We found that, in general, $\phi>\beta$, which supports a similar finding first reported for simulations in the river network of the Walnut Gulch basin, Arizona. Theoretical estimation of $\beta$ and $\phi$ in RSNs is a complex open problem. Therefore, using results for a simpler problem associated with the expected width function and expected hydrograph for an ensemble of RSNs, we give heuristic arguments for theoretical derivations of the scaling exponents $\beta^{(E)}$ and $\phi^{(E)}$ that depend on the Horton ratios for stream lengths and areas. These ratios in turn have a known dependence on the parameters of the geometric distributions of RSN generators. Good agreement was found between the analytically conjectured values of $\beta^{(E)}$ and $\phi^{(E)}$ and the values estimated by the simulated ensembles of RSNs and hydrographs. The independence of the scaling exponents $\phi^{(E)}$ and $\phi$ with respect
\end{abstract}

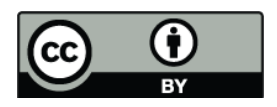

Correspondence to: R. Mantilla (ricardo-mantilla@uiowa.edu) to the value of flow velocity and runoff intensity implies an interesting connection between unit hydrograph theory and flow dynamics. Our results provide a reference framework to study scaling exponents under more complex scenarios of flow dynamics and runoff generation processes using ensembles of RSNs.

\section{Introduction}

Several theoretical and observational results have led to the development of a nonlinear geophysical theory of floods in river networks (Gupta et al., 2007). The central hypothesis of the theory states that solutions of coupled mass and momentum conservation equations under suitable physical parameterizations in a self-similar river network produce spatial power laws, or scaling relations, between peak flows and drainage area in the limit of large area. Scaling in peak flows is an emergent property that is common to many nonlinear geophysical systems (Lovejoy et al., 2009). A central objective of the theory, given a space-time rainfall field for a rainfall-runoff event, is to predict the values of the intercept and exponent in the power law relation from the physics of hillslope-link runoff generation and runoff transport, and test it against observed power law. The flood of June 2008 in Eastern Iowa is the most recent example that supports the validity of the main hypothesis of the theory over four orders of magnitude variation in drainage area (Gupta et al., 2010). Testing the scaling hypothesis using direct observations requires a large number of streamflow gauges in a river basin and data for multiple RF-RO events. Unfortunately, availability of such data sets is rather limited, which poses a great challenge for future development of the theory. In this paper,

Published by Copernicus Publications on behalf of the European Geosciences Union and the American Geophysical Union. 
we take the first step towards developing a multi-step diagnostic framework to understand the behavior of the powerlaw parameters, which can be compared to observations for developing suitable physical parameterizations.

Ogden and Dawdy (2003) conducted the first study in the $21 \mathrm{~km}^{2}$ Goodwin Creek experimental watershed (GCEW), and observed that the slopes and intercepts of the peakflow scaling, or power-law, relations vary from one event to the next. Furey and Gupta (2005) found that the eventto-event variability in the slopes and intercepts of the peakflow scaling relations is connected to rainfall-excess depth and duration for storms. Building on this finding, Furey and Gupta (2007) developed a diagnostic framework to predict slopes and intercepts of peak flow scaling functions in GCEW. Mantilla et al. (2006) demonstrated, for the $150 \mathrm{~km}^{2}$ Walnut Gulch basin in Arizona, that changes in the dynamics of runoff transport in the river network lead to changes in the value of the scaling exponent. Recently, Mandapaka et al. (2009) showed using numerical simulations in the $1100 \mathrm{~km}^{2}$ Whitewater basin, Kansas how different aspects of rainfall variability affect the scaling exponent. The results of these studies have already provided significant insights to our physical understanding of scaling in peak flows. However, the fact that these studies have been performed on specific individual river networks leaves open key questions about the role of self-similar topology of a river network in determining the value of the exponent and intercept. We address this issue here.

Gupta et al. (1996) first derived the scaling of peak flow versus drainage area in a self-similar Peano channel network. Their derivation was based on analysis of geometric properties of the network width function, which is defined to be the number of channel links as a function of distance from the outlet (Rodríguez-Iturbe and Rinaldo, 1997). Denoting $W_{\omega}(x)$ as the width function for a stream of Strahler order $\omega$, the scaling relationship with respect to the upstream contributing area $A_{\omega}$ follows from the fractal structure of the maximum contributing set and is given by $\max _{x} W_{\omega}(x)=$ $c_{\beta} A_{\omega}^{\beta}$. Here, $\beta$ and $c_{\beta}$ are constants that depend on the network topology. Under the assumptions of, (i) spatially uniform instantaneous inputs to the network, and (ii) movement through the network at a uniform velocity without attenuation, the streamflow hydrograph at the outlet of any sub-basin will have the same shape as the width function for that subbasin. Therfore, the scaling behavior of peak flows in the Peano network will be the same as that for the maximum of the width function. Troutman and Over (2001) generalized these results to a wide class of deterministic self-similar networks. Menabde et al. (2001) considered a more realistic physical situation by assuming that flow in a network is represented by a mass conservation equation (Gupta and Waymire, 1998), and undergoes attenuation due to a change in a channel storage under uniform flow velocity. They found for the Peano and the Mandelbrot-Vicsek networks that, as
$A \rightarrow \infty$, the peak flow obeys scaling, $\max _{t} h_{\omega}(t)=c_{\phi} A_{\omega}^{\phi}$. Here $h_{\omega}(t)$ is a flow hydrograph. They also observed that the exponent $\phi<\beta$. The above analysis was extended to the class of random self-similar networks (RSNs) with Bernoulli-distributed generators. Veitzer and Gupta (2000) introduced RSNs to represent self-similarity and randomness observed in real river networks. Menabde et al. (2001) found that $\phi<\beta$ in RSNs. Subsequently, Mantilla et al. (2006) simulated hydrographs in the Walnut Gulch basin, Arizona under the same set of physical assumptions that Menabde et al. (2001) had used. Surprisingly, the results showed that $\phi>\beta$. They conjectured that the qualitative different behavior between the two scaling exponents reflects the differences between the interplay of aggregation and attenuation of stream flows in a real network. Mantilla (2007) investigated the generality of this result using an ensemble of RSNs. His findings supported that $\phi>\beta$. Communication of this result is a major objective of our paper.

RSNs are constructed recursively from random generators, which are essentially simple order- 2 networks with a random number of interior nodes (Veitzer and Gupta, 2000). A distinction is made between interior and exterior generators, and the probability distribution can be different for these two types of generators (Troutman, 2005). The class of RSN models exhibit important topological features of real networks that other models, such as the random topology model (Shreve, 1966), or optimal channel networks (Rigon et al., 1993), do not. These properties include the Horton law of stream numbers (Veitzer and Gupta, 2000), Hack's law (Troutman, 2005), scaling properties of the width function (Veitzer and Gupta, 2001; Troutman, 2005), and the powerlaw tail probabilities of drainage areas (Veitzer et al., 2003). Tokunaga mean self-similar networks exhibit many properties of real networks, but do not include any statistical variability found in real networks (McConnell and Gupta, 2008). Building on an approach to estimate RSN generators for real networks that Troutman (2005) introduced, Mantilla et al. (2010) carried out an extensive analysis to estimate and test the properties of RSN generators for 30 river basins in different hydro-climatic regions of the United States. In all cases it was found that the RSN generators could be modeled by a geometric probability distribution.

In this paper we simulate RSNs using geometric distributed generators, and analyze the scaling properties of flow hydrographs that are obtained from solving mass and momentum conservation equations in each network (Gupta et al., 2007). Our objective is to test if $\phi>\beta$ holds under the assumption of constant flow velocity in space and time as Mantilla et al. (2006) originally reported for the Walnut Gulch basin, Arizona. Where possible, we compare our numerical results with the analytic expressions that Troutman (2005) obtained, which serves as benchmark for the accuracy of results from numerical simulations.

The rest of the paper is organized as follows. In Sect. 2 a detailed description of the RSN model and the process that is 
a)
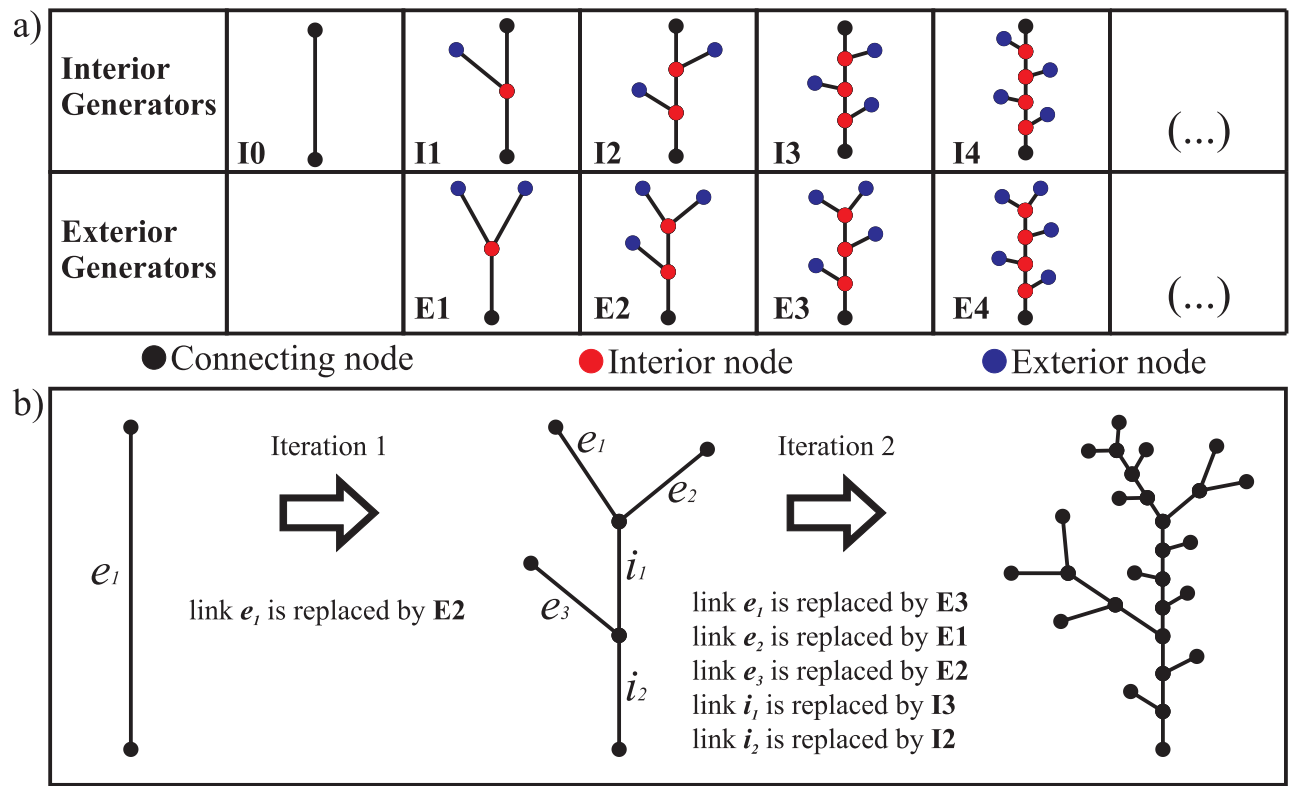

Fig. 1. (a) The populations of interior and exterior path-based generators. Generators are labeled by bold letters I (interior) and E (exterior) followed by an integer that represents number of interior nodes in the generator. (b) Two steps in the construction of an RSN indicating the replacements made.

used to create the ensemble of river networks are presented. In addition, the ensemble of flow hydrographs in RSNs that form the basis of this study are presented in this section. In Sect. 3 we use results from some members of the ensembles to shed light on the results that were obtained for simulation in the Walnut Gulch river basin. Later, in Sect. 4 we present a generalization of the results by analyzing the relation of exponents $\beta$ and $\phi$ on the full ensemble of RSNs. In Sect. 5 we present some new analytical results of scaling properties for RSNs and in Sect. 6 we test the validity of these results on our ensemble of river networks and flow hydrographs. Finally in Sect. 7 we present the conclusions of this work and some areas of future research.

\section{Generating RSN ensembles and flow routing}

RSNs are constructed by replacing, in an iterative fashion, all the links of a network by randomly sampled generators (Veitzer and Gupta, 2000). The process is initiated with a network that consists of only a single link, and this link is replaced with a randomly sampled generator. Then the links in the resulting network are all replaced with randomly sampled generators, and so on. Thus, at each step of the iteration process, the branching structure of the network becomes more complex. Each link replacement in the iterative process is done in a manner that depends on whether the link to be replaced is interior or exterior, where exterior links are defined as those with no upstream connecting links. Generators from one population, known as interior generators, replace interior

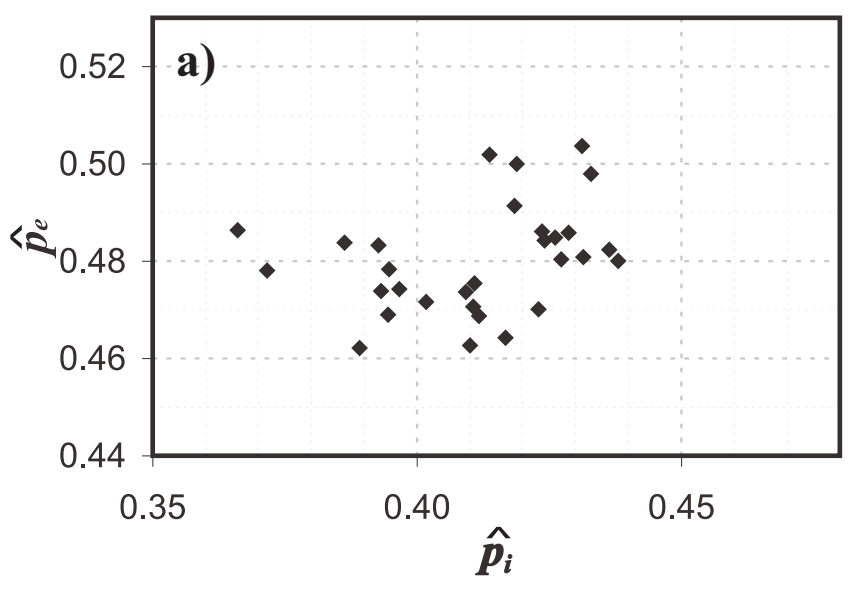

Fig. 2. (a) Parameter space determined by the estimated parameters $p_{\mathrm{i}}$ and $p_{\mathrm{e}}$ for generators sampled from different regions in the US.

links and exterior generators constituting a different population replace exterior links. Construction of RSNs thus requires specification of two probability distributions governing the random sampling of the different generator types, and all sampled generators are assumed to be mutually independent. Figure 1a illustrates the two populations of generators to be used in this paper and Fig. 1b illustrates two iterations of the replacement process.

Mantilla et al. (2010) demonstrated that the probability distribution of external and internal generators of real river networks follow geometric distributions given by 

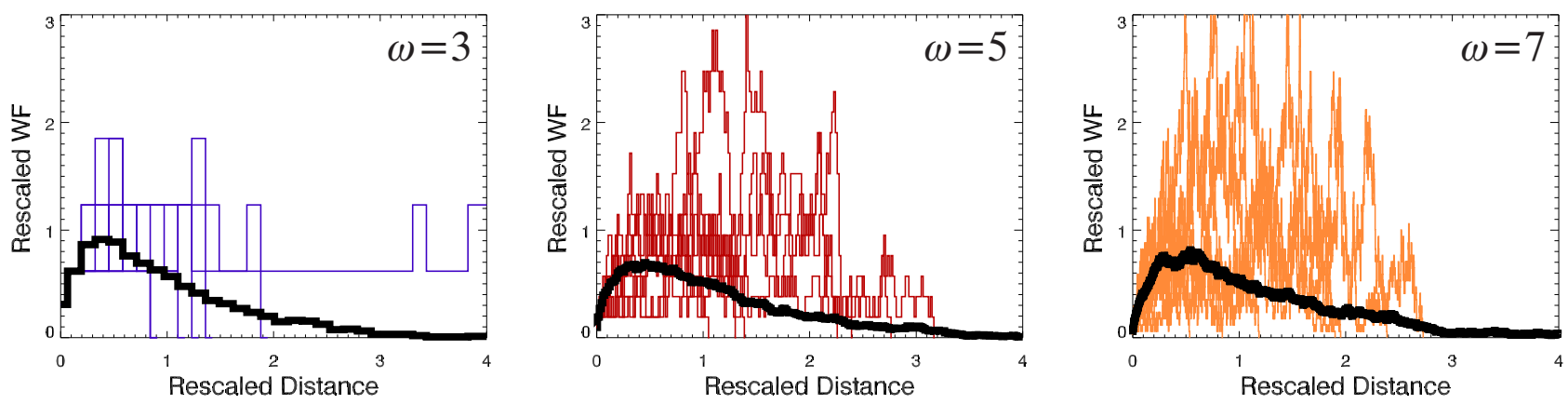

Fig. 3. Width functions, $W_{\omega}(x)$ and average width function $E\left[W_{\omega}(x)\right]$ (solid black line) for RSN with different order $\omega$, and parameters $p_{\mathrm{i}}=0.36$ and $p_{\mathrm{e}}=0.48$. The vertical axis (number of links at distance $x$ from the outlet) has been rescaled by the factor $\left(R_{\mathrm{C}} / R_{\mathrm{A}}\right)^{\omega-1}$ and the horizontal axis (distance from the outlet $x$ ) has been rescaled by the factor $R_{\mathrm{C}}^{\omega-1} \cdot R_{\mathrm{A}}$ and $R_{\mathrm{C}}$ are the Horton ratios of areas and lengths, respectively.
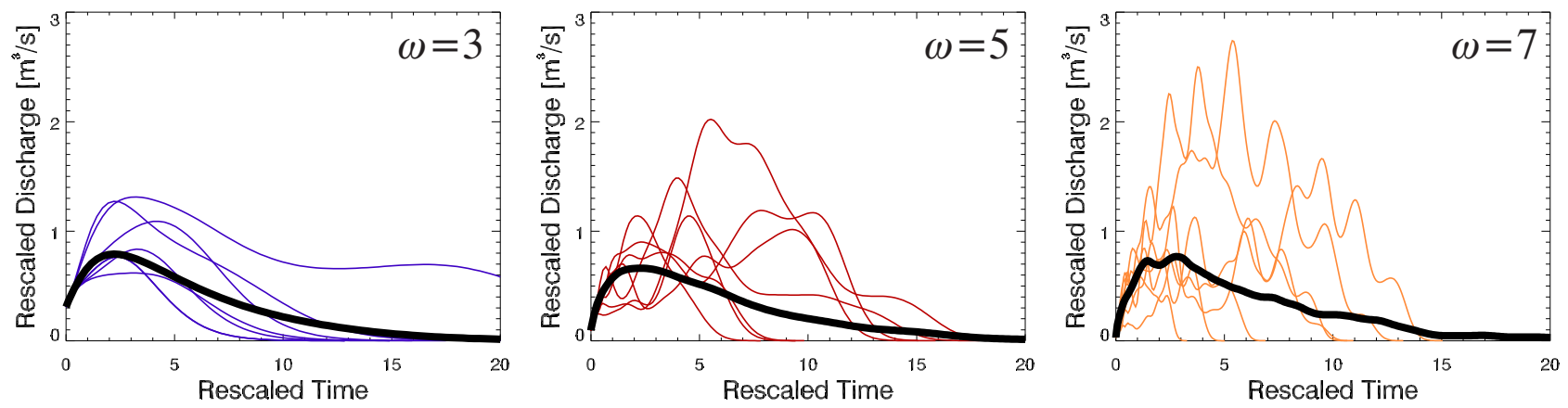

Fig. 4. Hydrographs, $h_{\omega}(t)$ and average hydrograph $E\left[h_{\omega}(t)\right]$ (solid black lines) for RSN with different order $\omega$, and parameters $p_{\mathrm{i}}=0.36$ and $p_{\mathrm{e}}=0.48$. The vertical axis has been rescaled by the factor $\left(R_{\mathrm{C}} / R_{\mathrm{A}}\right)^{\omega-1}$ and the horizontal axis has been rescaled by the factor $R_{\mathrm{C}}^{\omega-1}$, with $R_{\mathrm{A}}$ and $R_{\mathrm{C}}$ given by Eq. (1).

$P\left(K_{\mathrm{i}}=k_{\mathrm{i}}\right)=p_{\mathrm{i}}\left(1-p_{\mathrm{i}}\right)^{k_{\mathrm{i}}}, k_{\mathrm{i}} \geq 0$ for interior generators and $P\left(K_{\mathrm{e}}=k_{\mathrm{e}}\right)=p_{\mathrm{e}}\left(1-p_{\mathrm{e}}\right)^{k_{\mathrm{e}}-1}, k_{\mathrm{e}} \geq 1$ for exterior generators, where the random variable $K_{i}$ is the number of interior nodes in interior generators and the random variable $K_{\mathrm{e}}$ is the number of interior nodes in exterior generators (see Fig. 1 for node types). They found that the parameters $p_{\mathrm{i}}$ and $p_{\mathrm{e}}$ are not equal, but each is constant with respect to iteration step in the replacement process, indicating scale invariance in real networks. The geometric parameters are observed to exhibit significant basin-to-basin variability (Fig. 2).

We simulated ensembles of 1000 RSNs for combinations of $p_{\mathrm{i}}$ and $p_{\mathrm{e}}$ in the parameter space. A total of 35 combinations of the parameters in the range $p_{\mathrm{i}} \in[0.36,0.48]$ and $p_{\mathrm{e}} \in[0.45,0.53]$ were selected for a total of 35,000 distinct topologies. A sampling interval of 0.2 was deemed sufficient after performing several tests of sensitivity of topological scaling properties to the parameters $p_{\mathrm{i}}$ and $p_{\mathrm{e}}$. We arrived at the 1000 RSNs per combination by finding a balance between statistical accuracy of the estimations performed for the ensemble and computational limits to the number of numerical simulations that can be performed.
Troutman (2005), building on the analyses and results of Veitzer and Gupta (2000), has shown that, for the family of RSNs with geometrically distributed interior and exterior generators with parameters $p_{\mathrm{i}}$ and $p_{\mathrm{e}}$, the Horton ratios of areas and lengths $R_{\mathrm{A}}$ and $R_{\mathrm{C}}$, respectively, are given by,

$R_{\mathrm{A}}=\frac{p_{\mathrm{i}}+p_{\mathrm{e}}}{p_{\mathrm{i}} p_{\mathrm{e}}}$, and $\quad R_{\mathrm{C}}=\frac{1}{p_{\mathrm{i}}}$.

Note that Eq. (1) holds for the RSN model under the assumption of constant hillslope areas or independent and identically distributed random hillslope areas (Veitzer and Gupta, 2000).

Estimating scaling exponents requires sets of networks of different orders. This can be achieved in two ways: (i) by creating a large network of order $\Omega$ and then sampling width functions $W_{\omega}(x)$ at the end of the embedded complete order streams, or (ii) by creating networks of different orders $\omega=1,2, \ldots, \Omega$ and sampling the width function at the outlet of each individual river network. We choose approach (ii) because it eliminates the possibility of dependencies created by the nested structure of a single large order $\Omega$ network. In addition, it is easier to develop statistical tests when the sample size is equal for different order $\omega$ networks 
which are provided by approach (ii). Figure 3 shows the estimated $E\left[W_{\omega}(x)\right]$ (solid black line) for RSNs of different order $\omega$, with parameters $p_{\mathrm{i}}=0.36$ and $p_{\mathrm{e}}=0.48$. Figure 3 also shows a few of the sampled width functions $W_{\omega}(x)$.

For each of the members of the ensemble of RSNs, we calculated the flow hydrograph produced by applying an instantaneous-uniform runoff event over the entire network. Flow hydrographs are obtained by numerically solving, for each link in the river network, the link based flow equation,

$\frac{d q(t)}{\mathrm{d} t}=K(q(t))\left[a R(t)+q_{1}(t)+q_{2}(t)-q(t)\right]$

developed by Gupta and Waymire (1998) and Menabde and Sivapalan (2001), where $q(t)$ is the flow at time $t, K()$ is in general a non-linear function of $q(t), a$ is the total hillslope area, $R(t)$ is the runoff intensity coming from the hillslopes, and $q_{1}(t)$ and $q_{2}(t)$ are flows coming from the upstream tributaries when present. We assume that flow velocity is constant at all times, making $K(q(t))=v / l$ where $l$ is the link length (Menabde et al., 2001). We let $v=1 \mathrm{~m} \mathrm{~s}^{-1}$, $l=300 \mathrm{~m}$ and $a=0.1 \mathrm{~km}^{2}$ throughout the network. These values are consistent with observed properties of real river networks (Mantilla, 2007). $R(t)$ equals 0 for all $t$, and an initial condition $q(0)=1 \mathrm{~m}^{3} \mathrm{~s}^{-1}$ is imposed for all of the links in the network. Note that this initial condition is equivalent to the volume of water generated by a finite duration runoff event that is applied instantaneously from the hillslope to the nearest channel, for example a runoff intensity of $36 \mathrm{~mm} \mathrm{~h}^{-1}$ applied during $5 \mathrm{~min}$ or a runoff intensity of $180 \mathrm{~mm} \mathrm{~h}^{-1}$ applied during $1 \mathrm{~min}$. The stored water in the channel for the instantaneous event is $S=a i t_{\mathrm{s}}$, where $a$ is the total hillslope area, $i$ is the runoff intensity and $t_{\mathrm{s}}$ is the storm duration. Under the assumption that cross sectional area and flow depth are constant across the link we can write $q=\frac{v}{l} S$, and it follows that $i=\frac{q l}{t_{s} a v}$. A sample of the hydrographs calculated is shown in Fig. 4.

\section{Interpreting simulation results in RSNs corresponding to the Walnut Gulch, AZ}

Our first objective is to use simulated ensembles of hydrographs in RSNs corresponding to the Walnut Gulch basin, and develop a framework that allows us to test the validity of the results reported by Mantilla et al. (2006). We used the techniques that Mantilla et al. (2010) explained to estimate the parameters of the geometric distributions for the frequencies of the interior and the exterior generators in the Walnut Gulch basin. We found that the two distinct geometric distributions have parameters $p_{\mathrm{i}}=0.345$ and $p_{\mathrm{e}}=0.462$. The results are shown in Fig. 5, where confidence intervals for the values of $p_{\mathrm{i}}$ and $p_{\mathrm{e}}$ are also given.

In order to test the hypothesis $\phi>\beta$ for this type of networks we generated an ensemble of 1000 RSNs, and calculated the two scaling exponents for each individual network. Mantilla et al. (2006) used Horton ratios to calculate the scaling exponents as explained in detail in Sect. 4. Furey and Troutman (2008) have suggested an improved estimation technique for the Horton ratios involving geometric means rather than arithmetic means that is routinely used. However, we did not use this improved technique in our analysis so we could compare our results with Mantilla et al. (2006), but it is used in Sect. 4.

The estimated exponents exhibited a large degree of variability amongst the individual networks in the ensemble. The average value of $\beta$ is 0.460 with a standard deviation of 0.0067 , which is close to the value of 0.48 that Mantilla et al. (2006) obtained. Similarly, the average value for $\phi$ is 0.485 with standard deviation of 0.0095 , which is smaller than the value of 0.55 in Mantilla et al. (2006). The histogram for the values calculated are shown in Fig. 6 . The range of the simulated scaling exponents includes the values observed in the Walnut Gulch basin.

Although the hypothesis $\phi>\beta$ holds for the average values, it does not hold for every river network in the ensemble that was analyzed. In Fig. 7 plots comparing $\beta$ and $\phi$ are given. It can be seen that the hypothesis $\phi>\beta$ breaks down for some of the networks.

Our analysis of the data indicates that the hypothesis $\phi>$ $\beta$ holds for approximately 700 of the 1000 networks in the ensemble. The histogram of the difference is shown in Fig. 8.

The results in this section provide a context to understand scaling of flows in a more generic family of RSNs that Mantilla et al. (2010) have identified. This knowledge is necessary because only a handful of basins around the world are as heavily instrumented as the Walnut Gulch. Therefore, the understanding of these features can come from simulations or analytic work in the solution of the flow equations on RSNs. In the following section we focus our attention on how the two scaling exponents compare with each other, and address issues regarding estimation of scaling exponents in simulated RSN topologies that are larger than order 6 of the Walnut Gulch basin.

\section{Comparing two scaling exponents for peak flow prediction on RSN ensembles}

The goal of this section is to present the analysis of two scaling exponents $\beta$ and $\phi$ that are estimated for the set of RSNs with geometrically distributed generators and the corresponding flow hydrographs. Specifically, we test the hypothesis that the scaling peak flow exponent under constant velocity routing $\phi$ is larger than the scaling exponent of the peak of the width function $\beta$. As indicated in the introduction our hypothesis is qualitatively different from results obtained in idealized fractal river networks and for a family of RSNs with Bernoulli-distributed generators (Menabde et al., 2001). 
Interior Generators

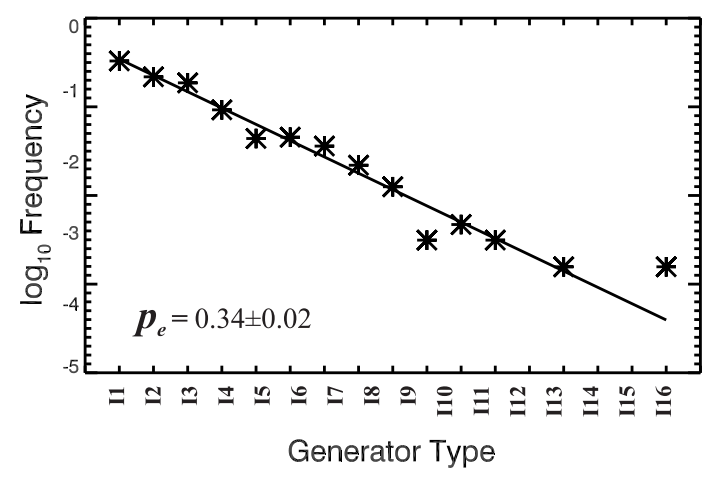

\section{Exterior Generators}

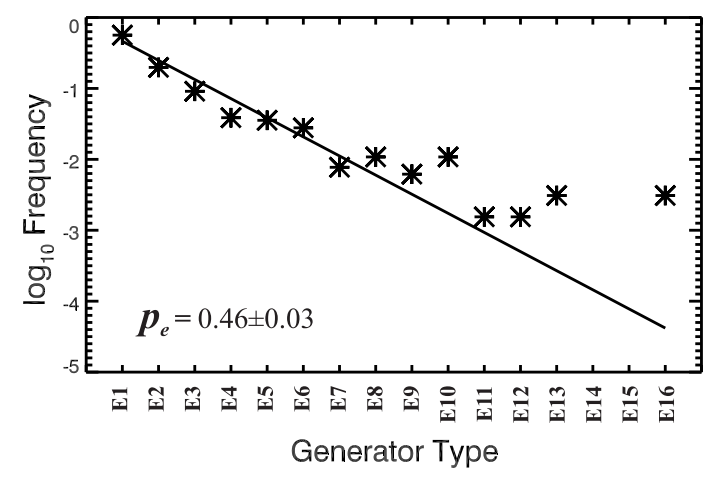

Fig. 5. Estimated parameters for a geometric distribution describing generators in Walnut Gulch, AZ.
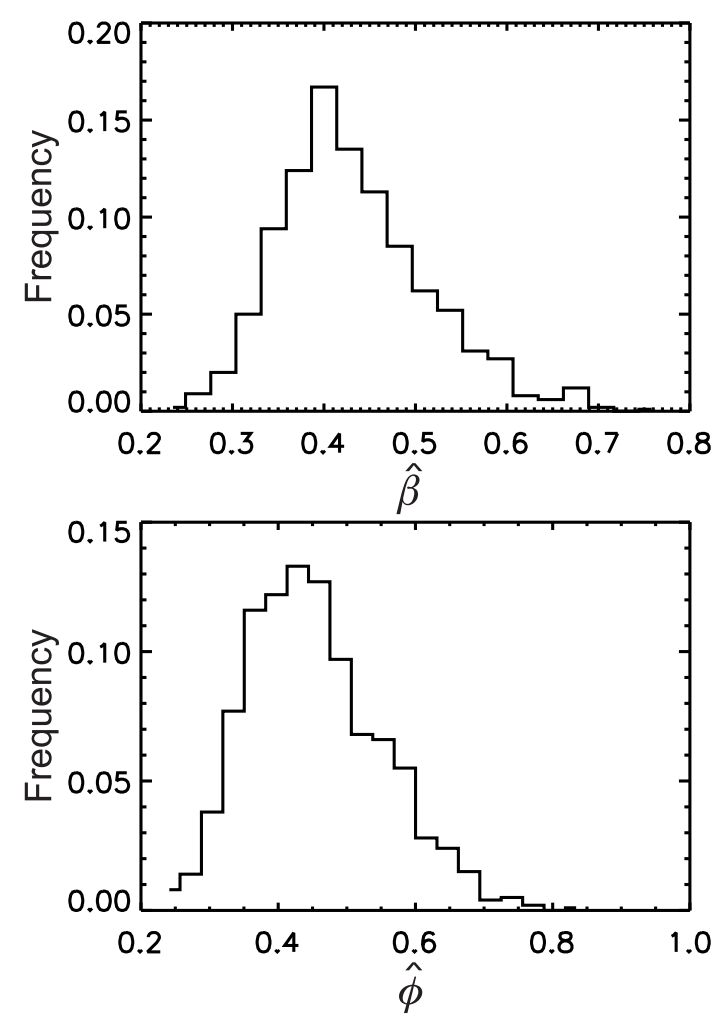

Fig. 6. Histograms for $\beta$ and $\phi$ for RSNs with parameter values $p_{\mathrm{i}}=0.345$ and $p_{\mathrm{e}}=0.462$.

\subsection{The scaling exponent $\beta$}

We use the ensemble of networks to obtain an estimate $\hat{\beta}$ of the parameter $\beta$ defined by,

$\beta=\log R_{\Theta} / \log R_{\mathrm{A}}$

where, $R_{\Theta}$ is the Horton ratio for the maximum of the width function $\Theta_{\omega}=\max _{x} W_{\omega}(x)$. The Horton ratio $R_{\Theta}$ is estimated as the linear least square regression slope between
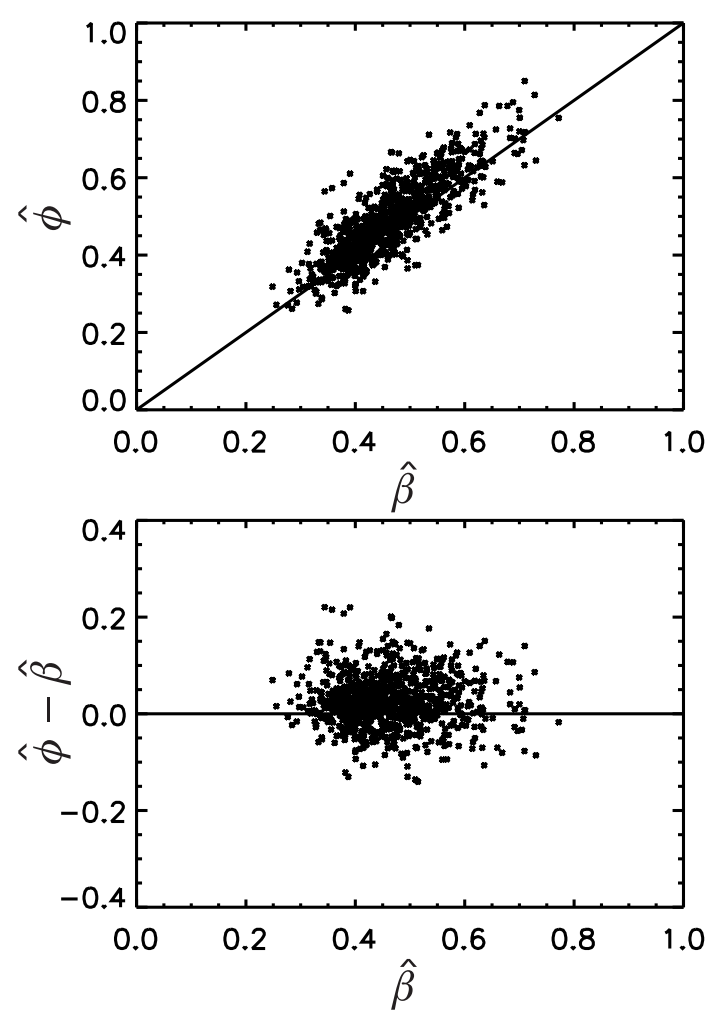

Fig. 7. Histograms for $\beta$ and $\phi$ for RSNs with parameter values $p_{\mathrm{i}}=0.345$ and $p_{\mathrm{e}}=0.462$.

the $E\left[\log \left(\Theta_{\omega}\right)\right]$ vs. stream order $\omega$ following recommendations by Furey and Troutman (2008). It leads to a geometric mean for the Horton ratio rather than the arithmetic mean that is routinely used. The parameter $\Theta_{\omega}$ has been studied by Veitzer and Gupta (2000) for RSNs and by Mantilla et al. (2006) for real networks as it has a direct bearing on the scaling relation for peak flows. The random variable $\Theta_{\omega}$ is of particular interest because it has been shown by Veitzer and Gupta (2001) to exhibit statistical self similarity (SSS); 


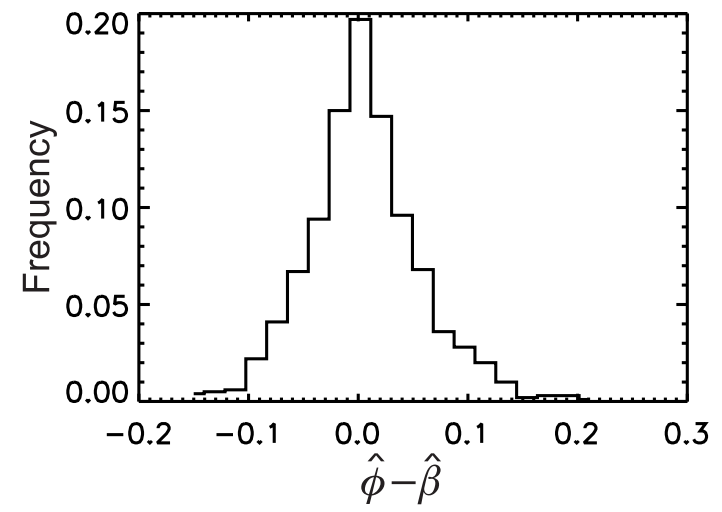

Fig. 8. Histogram of $\phi-\beta$.

which means that $\Theta_{\omega} / E\left[\Theta_{\omega}\right] \stackrel{d}{=} \Theta_{\omega+1} / E\left[\Theta_{\omega+1}\right]$. This condition is necessary to assert that $\max _{x} W_{\omega}(x)=c_{\beta} A_{\omega}^{\beta}$ when upstream areas $A_{\omega}$ obey SSS. Figure 9 shows the field of values estimated over the parameter space being considered here that is taken from Mantilla et al. (2010). It is interesting to note that the contours have a nearly vertical orientation, which means that a change in $p_{\mathrm{i}}$ has a greater effect on $\beta$ than $p_{\mathrm{e}}$. We conjecture that this feature comes from the property that peak of the width functions are dominated by interior generators rather than exterior generators.

In addition, Komogorov-Smirnov tests confirmed that $\Theta_{\omega} / E\left[\Theta_{\omega}\right] \stackrel{d}{=} Z$ for $\omega \geq 4$, where the distribution of $Z$ does not depend on $\omega$. Figure 10 illustrates this property for RSNs with parameters $p_{\mathrm{i}}=0.36$ and $p_{\mathrm{e}}=0.48$. The collapse of the rescaled cumulative distributions of the random variable $\Theta_{\omega} / E\left[\Theta_{\omega}\right]$ into a single common distribution follows from scale independence (Fig. 10a). The same feature is demonstrated for rescaled basin areas $A_{\omega} / E\left[A_{\omega}\right]$ (Fig. 10b). The convergence of the probability distribution of the quantity $A_{\omega} / E\left[A_{\omega}\right]$ was proved analytically by Veitzer and Gupta (2000) and the convergence result is also given by Trout$\operatorname{man}(2005)$.

\subsection{The scaling exponent $\phi$}

Using the results from the numerical solutions of Eq. (2) we calculate the peak flow for each member of the ensemble of RSNs. These simulations are used to estimate the exponent $\phi$ using the relation,

$\phi=\log R_{Q} / \log R_{\mathrm{A}}$

where $R_{Q}$ is the Horton ratio for peak flows. Heretofore the estimator of the exponent $\phi$ is labeled $\widehat{\phi}$. Results of this estimation are presented in Fig. 11. Again it is to be noted that a change in $p_{\mathrm{i}}$ has a greater effect on $\phi$ than $p_{\mathrm{e}}$ suggesting that peak flows are influenced more by the interior generators than exterior generators. Comparing the results in Fig. 11 with those in Fig. 9 it is observed that $\widehat{\phi}>\widehat{\beta}$ for

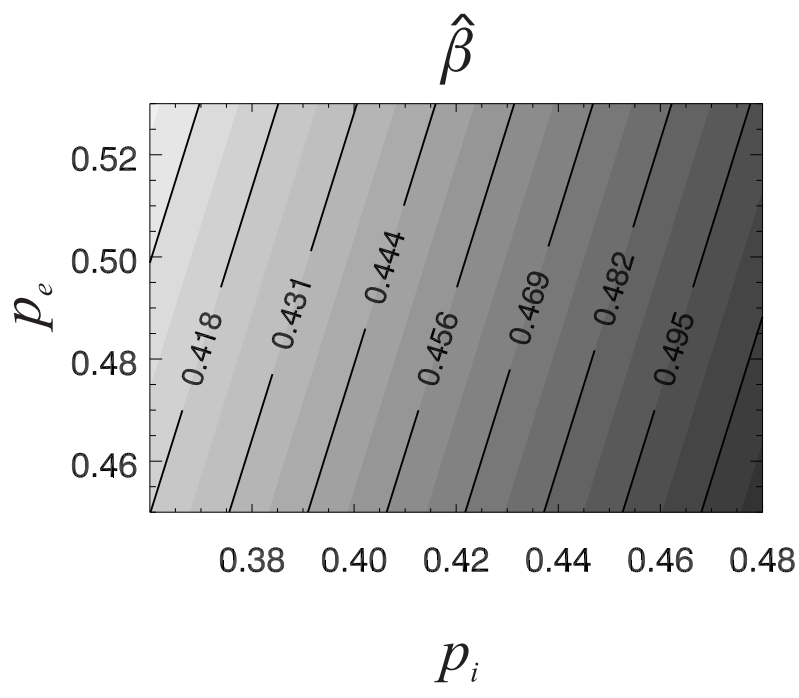

Fig. 9. Fitted plane to values for $\hat{\beta}$.

the range of parameters considered. Figure 12a shows an increasing value of the difference $\widehat{\phi}-\widehat{\beta}$ as a function of $\widehat{\beta}$. In the context of the Walnut Gulch basin, the observed difference $\phi-\beta=0.025$ as discussed in the last section, which is comparable to the value, $\widehat{\phi}-\widehat{\beta}=0.03$ shown in Fig. 12 .

SSS is also observed for rescaled peak flows (Fig. 13). ${ }^{1}$

\section{A summary of conjectures regarding two theoretical scaling exponents in RSN ensembles}

Developing theoretical formulas for the exponents $\phi$ and $\beta$ remains an open problem. However, recent developments in Troutman (2005) allow us to conjecture theoretical relations for the exponents $\beta^{(E)}$ and $\phi^{(E)}$ which are connected to the scaling properties of the ensemble average width function and hydrograph, respectively. The accuracy of the theoretical formulas is tested in Sect. 6 by comparing theoretical with estimated values from the ensembles of RSNs used in this study.

\subsection{On topologic network properties}

Define

$\beta^{(E)}=\frac{\log R_{\Theta}^{(E)}}{\log R_{\mathrm{A}}}$

where $R_{\Theta}^{(E)}$ is the Horton ratio for $\max _{x} E\left[W_{\omega}(x)\right]$ and $R_{\mathrm{A}}$ is the Horton ration of areas. Here $W_{\omega}(x)$ is the number of links

\footnotetext{
${ }^{1}$ We changed the value of the intensity of the event and we found that it does not have any effect on the value of the estimated exponents. Modifying the value of intensity is equivalent to rescaling the discharge axis by a constant. The same result was obtained when we double the flow velocity value. Modifying the velocity value is equivalent to rescaling the time axis by a constant.
} 

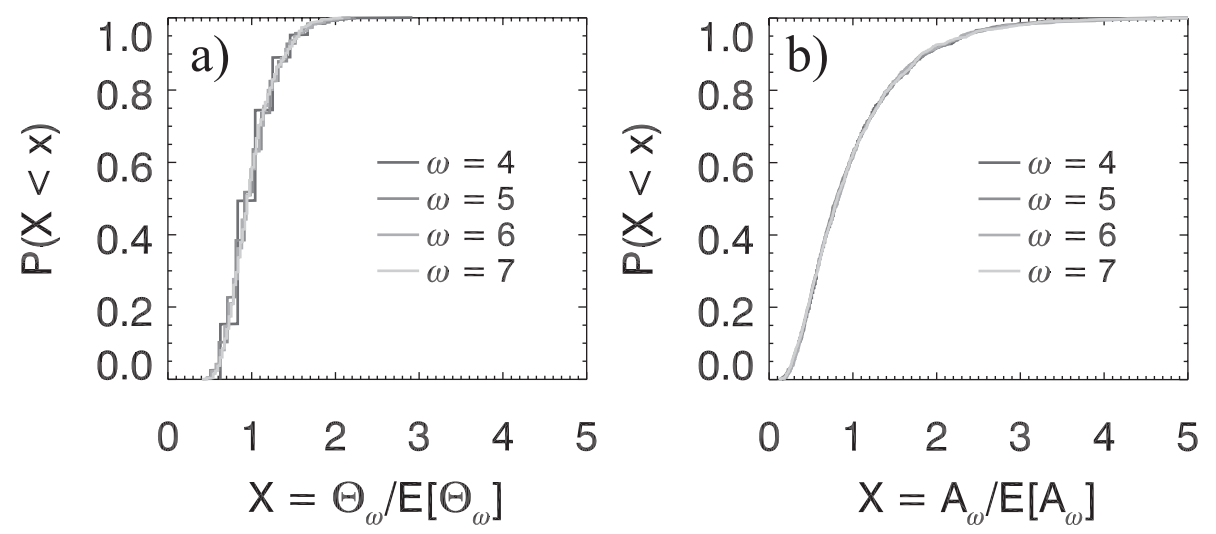

Fig. 10. The colapste of rescaled distributions indicates statistical self-similarity (SSS) in the (a) value of the maximum of the width function and (b) the distribution of basin areas.

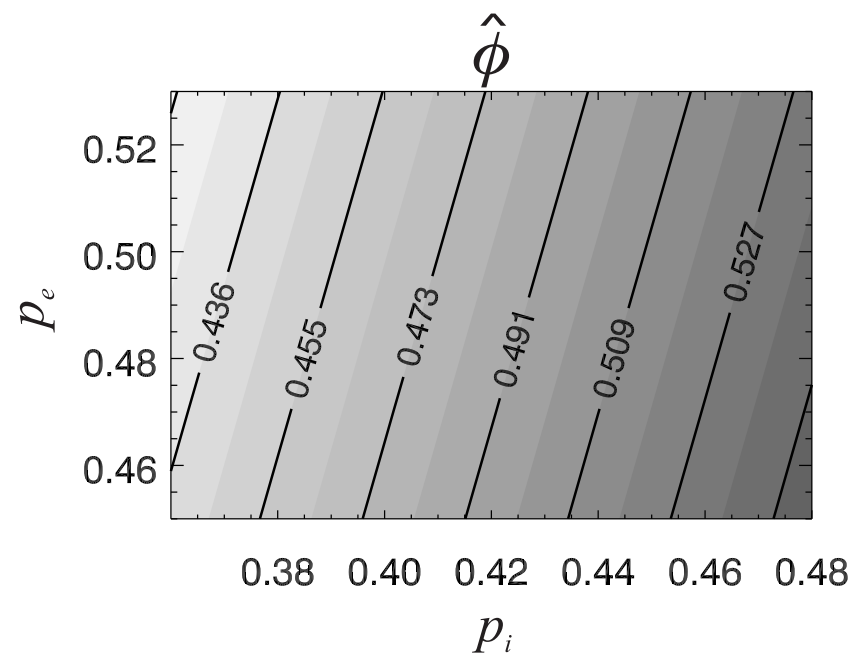

Fig. 11. Fitted plane to estimated $\hat{\phi}$.

having distance to the outlet equal to $x$. For RSNs, distance to the outlet of a given link is simply a count of the number of links between the downstream end of the given link and the outlet. Thus, $\beta^{(E)}$ represents the scaling exponent describing the power-law behavior of $\max _{x} E\left[W_{\omega}(x)\right]$ with respect to drainage area. Note that this scaling exponent is defined for the maximum of the ensemble average (expected) width function, which is different from the maxima of individual random width functions considered so far.

Troutman (2005) presents theoretical results which suggest that $\beta^{(E)}$ is given by

$\beta^{(E)}=1-\frac{\log R_{\mathrm{C}}}{\log R_{\mathrm{A}}}$

where $R_{\mathrm{C}}$ and $R_{\mathrm{A}}$ are the Horton ratios of channel length and area respectively. More precisely, the result proved in Troutman (2005) gave scaling of the cumulative width function for RSNs, but a derivation of the scaling exponent in Eq. (6) for

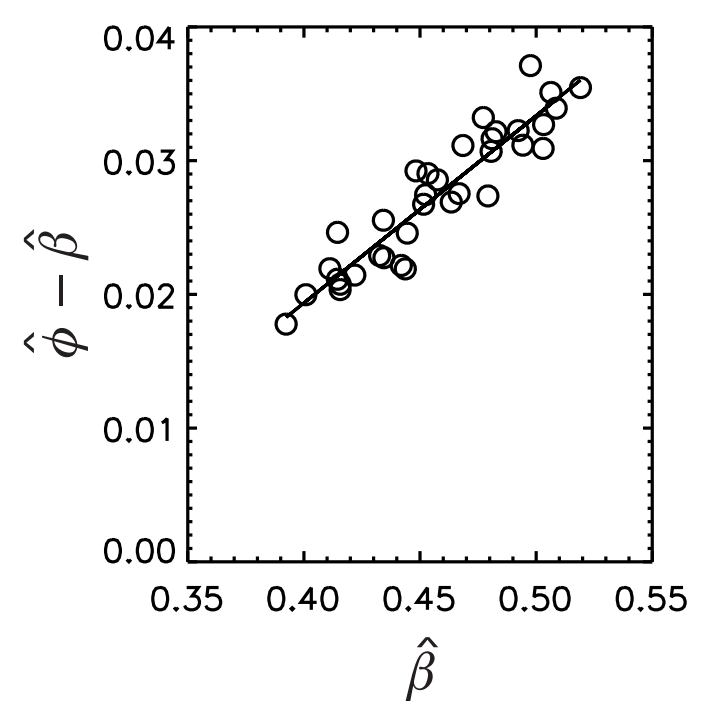

Fig. 12. Difference $\hat{\phi}-\hat{\beta}$ as a function of $\hat{\beta}$.

peak of the width function from the result for the cumulative width function remained open. In what follows we give a heuristic argument to support the conjecture in Eq. (6). The simulation results given in Sect. 4 provide further support that Eq. (6) is the correct expression for $\beta^{(E)}$ for RSNs.

Troutman (2005) proved that, for all $x \geq 0$,

$R_{\mathrm{A}}^{-\omega} E\left[J_{\omega}\left(R_{\mathrm{C}}^{\omega} x\right)\right] \rightarrow F(x) \quad$ as $\quad \omega \rightarrow \infty$

where

$J_{\omega}(x)=\sum_{j=0}^{\lfloor x\rfloor} W_{\omega}(j)$

is the cumulative width function, $\lfloor x\rfloor$ denotes the greatest integer less than or equal to $x$, and $F$ is a scale-independent function which is continuous and increasing in $x$, and which tends to a finite value as $x$ grows large. There are some technical restrictions on the generator distribution for Eq. (7) to 


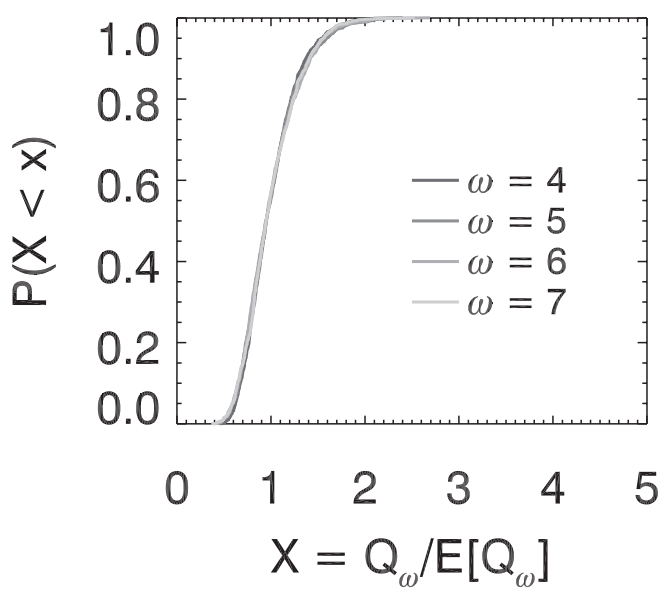

Fig. 13. The collapse of the distribution of rescaled peak flows for networks of different orders imply Statistical Self Similarity.

hold, but Troutman (2005) has shown that these restrictions are satisfied for geometric generators. Equation (7) is known as an "integral limit theorem" because it gives a result on the expected cumulative width function. An important open problem is to obtain what is known as a corresponding "local limit theorem" on the expected width function itself. We conjecture that such a result would have the form

$$
\left(R_{\mathrm{C}} / R_{\mathrm{A}}\right)^{\omega} E\left[W_{\omega}\left(\left\lfloor R_{\mathrm{C}}^{\omega} x\right\rfloor\right)\right] \rightarrow f(x) \quad \text { as } \quad \omega \rightarrow \infty
$$

The scaling on the left hand side is formally obtained by differentiating Eq. (7) with respect to $x$, although such an operation is not justified because $E\left[J_{\omega}(x)\right]$ is discontinuous in $x$ and therefore it is not differentiable. We would then expect the maximum of the expected width function to obey the same scaling, or

$\left(R_{\mathrm{C}} / R_{\mathrm{A}}\right)^{\omega} \max _{j} E\left[W_{\omega}(j)\right] \rightarrow$ const.

This would then lead to Eq. (6) because area scales as $R_{\mathrm{A}}^{\omega}$, implying

$\max _{j} E\left[W_{\omega}(j)\right] \sim \operatorname{const}\left(R_{\mathrm{A}}^{\omega}\right)^{1-\log R_{\mathrm{C}} / \log R_{\mathrm{A}}}$

Although a rigorous proof of Eqs. (9) and (10) remains open, the numerical results in Sect. 6 lend support to Eq. (6).

\subsection{On flows}

In this section we give asymptotic results that hold for linear flow in a RSN. The linear flow model to be used in this section is essentially the width function formulation of the geomorphological instantaneous unit hydrograph (GIUH) (Rodríguez-Iturbe and Rinaldo, 1997, Sect. 7.7). Very similar linear modeling approaches, but which are grid-based rather than link-based, are described in Olivera and Maidment (1999), Liu et al. (2003) and Olivera and Koka (2004). We shall assume here for simplicity that all rainfall excess is deposited into the upstream end of the link. But the asymptotic results below remain essentially the same if the lateral inflow is distributed over the length of the link. Let the time distribution of rainfall excess for an event be described by a function $R(t)$, which has integral one, and let total volume of rainfall excess for a link be denoted by $S_{\mathrm{R}}$. Under the linear flow model, it is assumed that the outflow from each link is the convolution of the total upstream inflow to the link with a link response function, say $g(t ; \Pi)$, where $\Pi$ is a vector of properties such as length and hydraulic parameters associated with the link. We may think of $g$ as a probability density function of the arrival time at the downstream end of a link of a unit parcel of water deposited instantaneously at the upstream end. The result is that the impulse response function for a path beginning at any initiating (upstream) link and consisting of $k$ connected links is the $k$-fold convolution of the individual link response functions. By linear superposition, the response function for the entire network is obtained by summing over all such initiating links in the network. In this section we assume that $\Pi, R(t)$, and $S_{\mathrm{R}}$ are spatially constant for all links in the network. This assumption allows us to collect terms in the sum over links in the network so that paths of a given length are weighted by the value of the width function at that length. As we shall see below, constancy of $\Pi$ is consistent with the constant velocity assumption of this paper.

We note here several well-known special cases. First, translation routing would result if $\Pi$ is a single element $T$ representing constant travel time through a link and $g$ is a delta function $g(t ; \boldsymbol{\Pi})=g(t ; T)=\delta(t-T)$. Another common model is the linear diffusion model for which the parameter $\Pi$ consists of link length $l$, celerity $C$, and diffusivity $D$. In this case $g$ is given by

$g(t ; T)=g(t ; l, C, D)=\frac{l}{\sqrt{4 \pi D t^{3}}} e^{-(l-C t)^{2} / 4 D t}$

Finally, for linear storage routing, for which simulation results are given in Sect. 4 , the link parameter $\Pi$ consists of a single constant element, $K$, and $g$ is defined to be the exponential probability density function

$g(t ; \boldsymbol{\Pi})=g(t ; K)=K e^{-K t}$

One important characteristic of $g$ is the mean residence time in a link, given by

$\bar{t}=\int_{0}^{\infty} \operatorname{tg}(t ; \mathbf{\Pi}) \mathrm{d} t$

For the three special cases, $\bar{t}$ is given by $T, l / C$, and $1 / K$, respectively.

For a randomly generated order- $\omega$ network from the RSN model, let $h_{\omega}(t)$ be flow at the outlet and denote the width function by $W_{\omega}(x), 0 \leq x<\infty$ (the width function is zero for $x$ larger than the topological mainstream length of the 
network). Then under our linear flow assumption, the network instantaneous unit hydrograph $q_{\omega}(t)$ is given by

$q_{\omega}(t)=\sum_{j=0}^{\infty} W_{\omega}(j) g_{j}(t ; \boldsymbol{\Pi})$

where $g_{j}=g * g * \ldots * g$ is the $(j+1)$-fold convolution of $g$ and $h_{\omega}(t)$ is given by $S_{\mathrm{R}}\left(R * q_{\omega}\right)(t)$. This method of expressing flow in terms of the width function is given in a number of publications in the context of GIUH (see for example Mesa and Mifflin, 1986; Marani et al., 1991; Rinaldo and Rodríguez-Iturbe, 1996 and Rodríguez-Iturbe and Rinaldo, 1997, Eq. 7.112). Next, let $V_{\omega}(t)$ be the corresponding cumulative flow, or

$V_{\omega}(t)=\int_{0}^{t} h_{\omega}(\tau) d \tau$

Using Eq. (15) we can obtain a convergence result analogous to Eq. (7) for cumulative flow; for every $x \geq 0$

$\left(S_{\mathrm{R}} R_{\mathrm{A}}^{\omega}\right)^{-1} E\left[V_{\omega}\left(R_{\mathrm{C}}^{\omega} \bar{t} x\right)\right] \rightarrow F(x) \quad$ as $\quad \omega \rightarrow \infty$

where $F$ is the same function as that on the right in Eq. (7). The proof of this result is very much like that given in Troutman and Karlinger (1988) for topologically random networks and will not be repeated here.

We point out several interesting features of the result in Eq. (17). First, the only property of $g$ that is of importance asymptotically is the mean residence time $\bar{t}$. (If $l$ is link length, we may define velocity to be $v=l / \bar{t}$.) Secondly, the form of the rainfall excess time distribution $R(t)$ does not enter into this result because letting the order of the network grow large makes this time distribution unimportant. Thirdly, and most important for this paper, the scaling with respect to order $\omega$ in Eq. (17) is the same as that of the expected cumulative width function in Eq. (7). However, we note that the result is again for cumulative hydrographs rather than hydrograph peaks, and further analysis would be necessary to obtain rigorously the result for peaks. Using an argument similar to that above for the width function, we conjecture that the scaling exponent for peaks would be

$\phi^{(E)}=1-\frac{\log R_{\mathrm{C}}}{\log R_{\mathrm{A}}}$

and that this would hold exactly and generally for any linear flow model under the restrictions imposed above. Simulations in Sect. 4 indicate that Eq. (18) does hold in the case of linear storage routing.

Equation (1) provides closed analytic expressions to calculate explicit formulas for $\beta^{(E)}$ and $\phi^{(E)}$ as a function of $p_{\mathrm{i}}$ and $p_{\mathrm{e}}$. Applying the formulas to the values that we determine to be our parameter space, produces the fields shown in Fig. 14. Notice that the contour lines are almost parallel to each other, implying that a plane can closely approximate the functional relation between the exponents $\beta^{(E)}$ and $\phi^{(E)}$ and the RSN model parameters $p_{\mathrm{i}}$ and $p_{\mathrm{e}}$ over our domain.

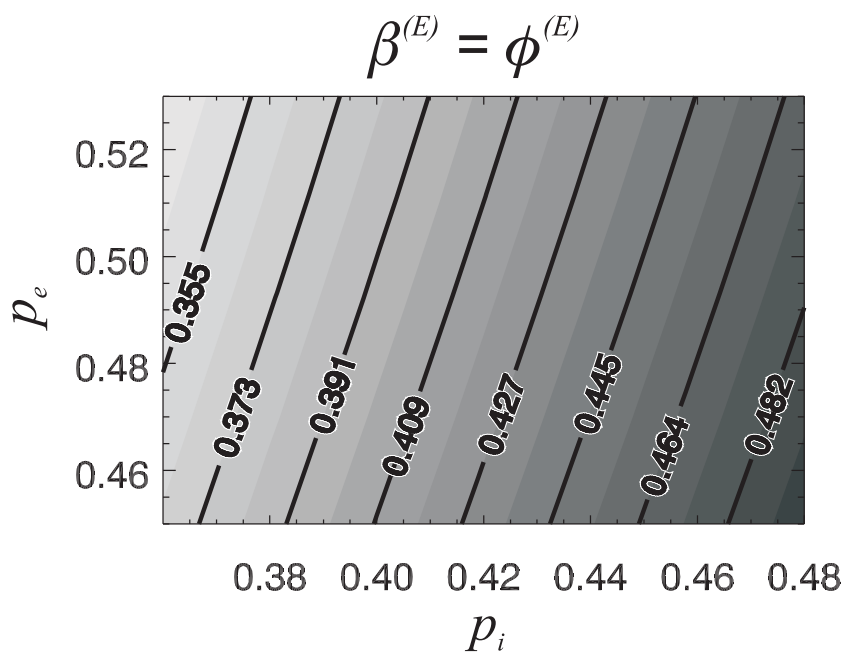

Fig. 14. Theoretical values for $\beta^{(E)}=\phi^{(E)}$ as functions of RSN parameters.

\section{Estimation of two theoretical scaling exponents in RSN ensembles}

\subsection{The scaling exponent $\beta^{(\mathrm{E})}$}

For every scale $\omega$ we estimate the maximum of the mean width function; labeled $\hat{\Theta}_{\omega}^{(E)}$. Horton ratios have been shown to hold for this quantity in an asymptotic sense (Troutman, 2005) which implies that,

$\frac{\Theta_{\omega+1}^{(E)}}{\Theta_{\omega}^{(E)}} \rightarrow R_{\Theta(E)} \quad$ as $\quad \omega \rightarrow \infty$

This result implies that estimates of $\hat{R}_{\Theta^{(E)}}$ can be obtained by taking the ratio of the estimated $\hat{\Theta}_{\omega}^{(E)}$ for networks with large $\omega$. Our largest simulated networks are of order $\Omega=7$. We found that the estimates of $\hat{R}_{\Theta(E)}$ were strongly biased by small variations of the values $\hat{\Theta}_{\omega}^{(E)}$. In order to eliminate this source of error we found that it is convenient to take the average ratio for the four largest orders. Thus, we define $\hat{R}_{\Theta}{ }^{(E)}$ in terms of the average ratio given by,

$\hat{R}_{\Theta(E)}=\frac{1}{3} \sum_{\omega=4}^{6}\left[\frac{\hat{\Theta}_{\omega+1}^{(E)}}{\hat{\Theta}_{\omega}^{(E)}}\right]$

In a similar fashion we use Eq. (5) to calculate $\hat{\beta}^{(E)}$. Figure 15 a shows the difference between the estimated values of the exponents and the theoretical value, $\beta^{(E)}$, computed by Eq. (6) for some combinations of $p_{\mathrm{i}}$ and $p_{\mathrm{e}}$ in our parameter space. We have fitted a plane passing through all those points using linear regression (Fig. 15b), and calculated the error with respect to the theoretical Eq. (18) (see Fig. 15c).

The average value of the difference $\beta^{(E)}-\hat{\beta}^{(E)}$ is 0.0047 , and the standard deviation is $3.9 \times 10^{-4}$. This difference is 
a)

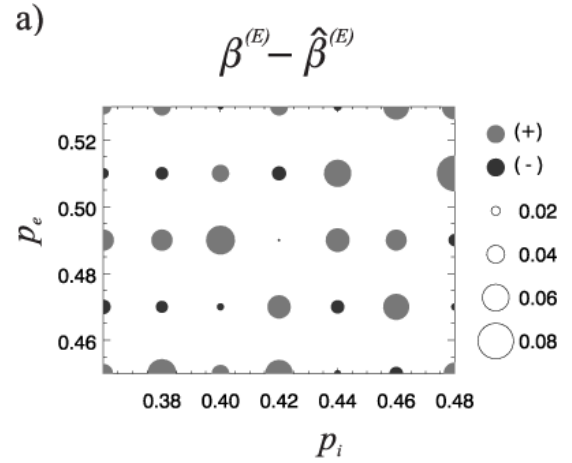

b)

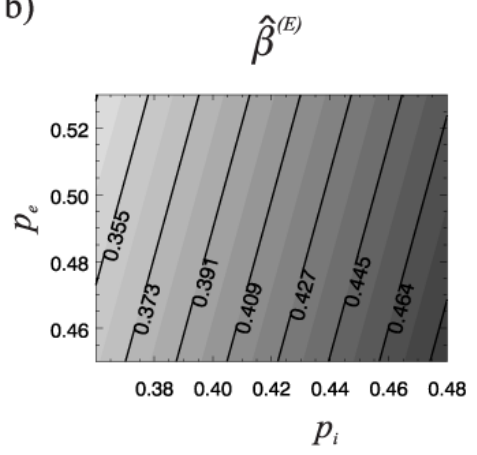

c)

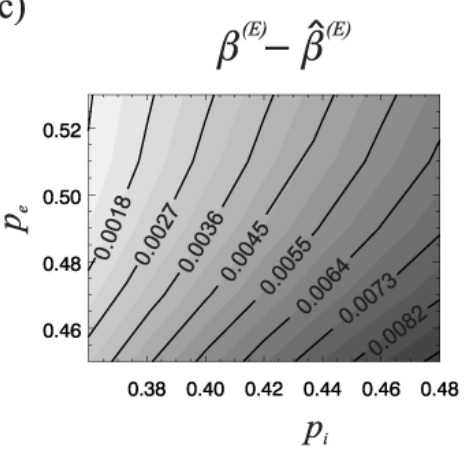

Fig. 15. Local error for estimated values, fitted plane and error of the fitted plane for the scaling exponents $\hat{\beta}^{(E)}$.

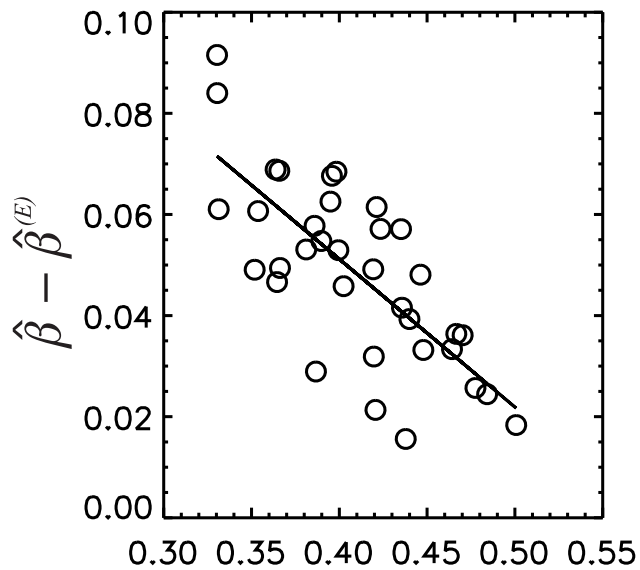

$\hat{\beta}^{(E)}$

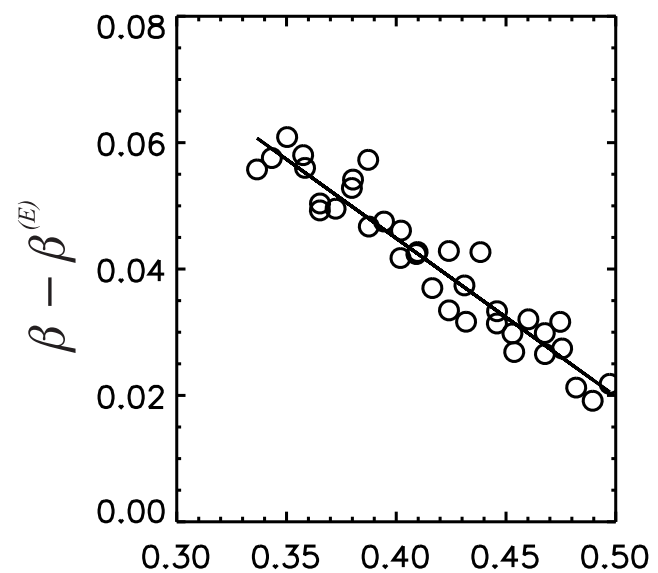

$\beta^{(E)}$

Fig. 16. (a) Difference $\hat{\beta}-\hat{\beta}^{(E)}$ as a function of $\hat{\beta}^{(E)}$ and (b) difference $\hat{\beta}-\beta^{(E)}$ as a function of $\beta^{(E)}$.

not statistically significant different from zero (5\% significance level). Figure $15 \mathrm{c}$ shows that the larger differences between the estimated and the theoretical exponents occur for larger values of $\beta^{(E)}$ however this departure can be attributed to the plane-fitting process since no systematic differences are observed in Fig. 15a. In addition, it is important to recall that the theoretical result holds asymptotically and the networks may not be large enough to achieve a perfect match.

We find that the difference $\hat{\beta}-\hat{\beta}^{(E)}$ has mean 0.074 and standard deviation $5.5 \times 10^{-4}$. In addition we found that the value of this difference exhibits a strong dependence on the estimated and theoretical exponents $\hat{\beta}^{(E)}$ and $\beta^{(E)}$. Figure 16a and 16b show that the largest deviations are observed for values of $\hat{\beta}^{(E)}$ and $\beta^{(E)}$ near 0.3 , while for values near 0.5 the difference is closer to zero. Note that the largest deviations are 8 times larger than the bias in estimation of the scaling exponents. Also note from this figure that the overall range of $\hat{\beta}^{(E)}$ values is greater than the overall range of $\hat{\beta}$ values. These differences are significant because the exponent $\beta$ has practical implication for flood predictions (Veitzer and Gupta, 2001; Mantilla et al., 2006).

\subsection{The Scaling exponent $\phi^{(E)}$}

A fitted plane for the estimated quantities is shown in Fig. 17a. The flow Eq. (2) is equivalent to a linear flow model defined in Sect. 5.2 with exponential link response function in Eq. (13). Therefore, it is conjectured that an exact expression for the exponent $\hat{\phi}^{(E)}$ is given by Eq. (18). We can calculate the difference between the theoretical expression and $\hat{\phi}^{(E)}$ (Fig. 17b). The average difference $\phi^{(E)}-\hat{\phi}^{(E)}$ is -0.0031 . The bias is similar to the one encountered for the parameters of the width function in the previous section.

An interesting result is that the bias observed in estimated scaling parameters of the max of the mean width function is the same as the one found for the scaling parameters of the max of the mean hydrograph. The average value of the difference $\hat{\beta}^{(E)}-\hat{\phi}^{(E)}$ is -0.0016 which is not statistically 

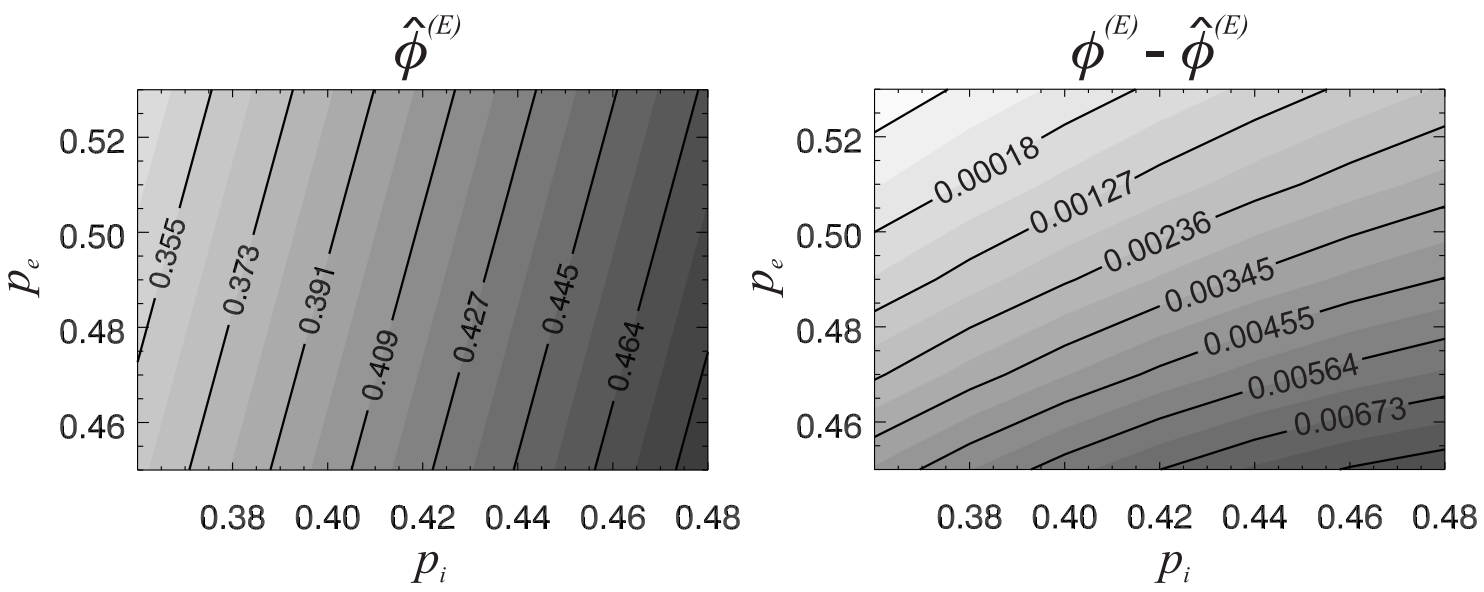

Fig. 17. (a) Fitted plane to estimated for $\hat{\phi}^{(E)}$ for the constant link and hillslope areas case. (b) Magnitude of the error for $\phi^{(E)}-\hat{\phi}^{(E)}$.

different from 0 with a $5 \%$ significance level. This result implies that the purely topologic exponent $\hat{\beta}^{(E)}$ is an accurate surrogate for the exponent $\hat{\phi}^{(E)}$ which represents the dynamical response of flows to runoff input in the network.

\section{Conclusions}

In order to understand the role of statistical self-similar topology observed in real river networks on spatial scaling statistics of hydrographs, we created Monte Carlo generated ensembles of 1000 RSNs with geometrically distributed interior and exterior generators with parameters $p_{\mathrm{i}}$ and $p_{\mathrm{e}}$, respectively (Mantilla et al., 2010). We calculated hydrographs in each of these networks by numerically solving the linkbased mass and momentum conservation Eq. (2) under the assumption of constant flow velocity. From these simulated networks and hydrographs the scaling parameters $\beta$ and $\phi$ were estimated. Results showed that, for RSNs with geometrically distributed generators, the scaling exponent $\phi>\beta$, which supports the finding that Mantilla et al. (2006) first reported for the Walnut Gulch basin. However, the use of ensembles of RSNs enabled us to find that the value of $\beta$ is a lower bound for the value of the exponent $\phi$. This finding has important implications for flood prediction in real networks that are ungauged.

Theoretical estimation of $\beta$ and $\phi$ in RSNs is a complex mathematical problem that remains open. However, progress has been made on a simpler problem which is the theoretical derivation of the scaling exponents $\beta^{(E)}$ and $\phi^{(E)}$ associated with the expected width function and expected hydrograph, respectively. Using rigorous asymptotic scaling results that Troutman (2005) obtained, we conjectured expressions for the functional dependence of $\beta^{(E)}$ and $\phi^{(E)}$ on the Horton ratios $R_{\mathrm{C}}$ and $R_{\mathrm{A}}$. These ratios in turn have a known dependence on the parameters of the geometric distributions $p_{\mathrm{i}}$ and $p_{\mathrm{e}}$.
Good agreement was found between the analytically conjectured values of $\beta^{(E)}$ and $\phi^{(E)}$ and the values estimated by the generated RSNs and hydrographs. Good agreement was also found for other geomorphic characteristics such as the scaling statistics of areas and SSS of maxima of width function. These results lend support to the correctness of the conjectured expressions in Eqs. (6) and (18). Our conclusions apply to the parameter space established in Mantilla et al. (2010) for real networks.

It was found that the exponents $\phi^{(E)}$ and $\phi$ are different and that $\phi>\phi^{(E)}$. This difference seems to be a direct consequence of the differences observed between $\beta$ and $\beta^{(E)}$. These differences in the exponents highlight the need to develop analytic results for the exponents $\beta$ and $\phi$, which have direct bearing in flood prediction for individual events (Mantilla et al., 2006).

The independence of the scaling exponents $\phi^{(E)}$ and $\phi$ with respect to the value of flow velocity and runoff intensity implies an interesting connection between unit hydrograph theory and flow dynamics. These results make the unit hydrograph theory fully consistent with the flow dynamics pertaining to constant velocity. Thus, a numerical solution of the link equation of flow can be interpreted as producing a family of GIUHs corresponding to the width function for each node in the network. Moreover, an average GIUH that solely depends on network Strahler order (e.g. Rinaldo and Rodríguez-Iturbe, 1996) is insufficient to describe flow transport in a river network. This results provide a reference framework to study scaling exponents under more complex scenarios of flow dynamics (Mantilla, 2007) and runoff generation processes (Furey and Gupta, 2007) using ensembles of RSNs. It also highlights the importance of combining numerical solutions of the equations along with analytic developments in order to make progress on this complex mathematical and scientific problem. 
Our results open several questions regarding the correct estimation of the scaling exponents. As shown by Furey and Troutman (2008) the estimation technique and preasymptotic effects can impact the correct estimation of the scaling exponents from ensembles. Thus, all our conclusions are subject to refinements as simulation of larger networks and larger ensembles become computationally possible, or as new analytic results become available.

\section{Appendix A}

\section{Notation}

$$
\begin{array}{ll}
A & =\text { Basin Area } \\
\omega & =\text { Strahler stream order } \\
W_{\omega}(x) & =\text { Width function of a stream of order } \omega \\
h_{\omega}(t) & =\text { Hydrograph of a stream of order } \omega \\
\Theta_{\omega} & =\text { maximum of width function } \max _{x} W_{\omega}(x) \\
Q_{\omega} & =\text { maximum of hydrograph } \max _{t} h_{\omega}(t) \\
\beta & =\text { Scaling exponent of } E\left[\Theta_{\omega}\right] \text { with respect to } A \\
\phi & =\text { Scaling exponent of } E\left[Q_{\omega}\right] \text { with respect to } A \\
\Theta_{\omega}^{(E)} & =\text { maximum of mean width function } \max _{x} E\left[W_{\omega}(x)\right] \\
Q_{\omega}^{(E)} & =\text { maximum of mean hydrograph } \max _{t} E\left[h_{\omega}(t)\right] \\
\beta^{(E)} & =\text { Scaling exponent of } \Theta_{\omega}^{(E)} \text { with } \operatorname{respect} A \\
\phi^{(E)} & =\text { Scaling exponent of } Q_{\omega}^{(E)} \text { with respect } A \\
i & =\text { Runoff intensity } \\
d & =\text { Runoff duration } \\
t_{\mathrm{c}} & =\text { Network concentration time }
\end{array}
$$

Acknowledgements. This research was partially supported by grants from the National Science Foundation to the University of Colorado and the University of Iowa. This support is gratefully acknowledged. Our thanks to the reviewers of this manuscript for providing insightful comments that helped improve the presentation of the ideas. We also thank John Moody and Lauren Hay of the USGS for helpful reviews, which improved the manuscript.

Edited by: Q. Cheng

Reviewed by: E. Foufoula and another anonymous referee

\section{References}

Furey, P. and Gupta, V. K.: Effects of excess rainfall on the temporal variability of observed peak discharge power laws, Adv. Water Resour., 28, 1240-1253, 2005.

Furey, P. R. and Gupta, V. K.: Diagnosing peak-discharge power laws observed in rainfall-runoff events in Goodwin Creek experimental watershed, Adv. Water Resour., 30, 2387-2399, 2007.

Furey, P. R. and Troutman, B. M.: A consistent framework for Horton regression statistics that leads to a modified Hack's law, Geomorphology, 102, 603-614, 2008.

Gupta, V. K. and Waymire, E. C.: Spatial variability and scale invariance in hydrologic regionalization, in: Scale dependence and scale invariance in hydrology, edited by: Sposito, G., Cambridge University Press, Cambridge, UK, 88-135, 1998.
Gupta, V. K., Castro, S. L., and Over, T. M.: On scaling exponents of spatial peak flows from rainfall and river network geometry, J. Hydrol., 187, 81-104, 1996.

Gupta, V. K., Troutman, B. M., and Dawdy, D. R.: Towards a Nonlinear Geophysical Theory of Floods in River Networks: An overview of 20 years of progress, in: Nonlinear Dynamics in Geosciences, edited by: Tsonis, A. A. and Elsner, J. B., Springer, New York, NY, 121-151, 2007.

Gupta, V. K., Mantilla, R., Troutman, B. M., Dawdy, D., and Krajewski, W. F.: Generalizing a nonlinear geophysical flood theory to medium-sized river networks, Geophys. Res. Lett., 37, L11402, doi:10.1029/2009GL041540, 2010.

Liu, Y. B., Gebremeskel, S., Smedt, F. D., Hoffmann, L., and Pfister, L.: A diffusive transport approach for flow routing in GIS-based flood modeling, J. Hydrol., 283, 91-106, doi:10.1016/S00221694(03)00242-7, 2003.

Lovejoy, S., Agterberg, F., Carsteanu, A., Cheng, Q., Davidsen, J., Gaonac'h, H., Gupta, V., L'Heureux, I., Liu, W., Morris, S. W. Sharma, S., Shcherbakov, R., Tarquis, A., Turcotte, D., and Uritsky, V.: Nonlinear Geophysics: Why We Need It, Eos Trans. AGU, 90, doi:10.1029/2009EO480003, 2009.

Mandapaka, P. V., Krajewski, W. F., Mantilla, R., and Gupta, V. K.: Dissecting the effect of rainfall variability on the statistical structure of peak flows, Adv. Water Resour., 32(10), 1508-1525, doi:10.1016/j.advwatres.2009.07.005, 2009.

Mantilla, R.: Physical Basis of Statistical Scaling in Peak Flows and Stream Flow Hydrographs for Topologic and Spatially Embedded Random Self-similar Channel Networks, Ph.D. thesis, University of Colorado, Boulder, CO, 2007.

Mantilla, R., Gupta, V. K., and Mesa, O.: Role of coupled flow dynamics and real network structures on Hortonian scaling of peak flows, J. Hydrol., 322, 155-167, 2006.

Mantilla, R., Troutman, B. M., and Gupta, V. K.: Testing statistical self-similarity in the topology of river networks, J. Geophys. Res., 115, F03038, doi:10.1029/2009JF001609, 2010.

Marani, A., Rigon, R., and Rinaldo, A.: A note on fractal channel networks, Water Resour. Res., 27, 3041-3049, 1991.

McConnell, M. and Gupta, V. K.: A proof of the Horton law of stream numbers for the Tokunaga model of river networks, Fractals, 16, 227-233, 2008.

Menabde, M. and Sivapalan, M.: Linking space-time variability of rainfall and runoff fields on a river network: A dynamic approach, Adv. Water Resour., 24, 1001-1014, 2001.

Menabde, M., Veitzer, S., Gupta, V. K., and Sivapalan, M.: Tests of peak flow scaling in simulated self-similar river networks, Adv. Water Resour., 24, 991-999, 2001.

Mesa, O. and Mifflin, E.: Scale Problems in Hydrology, On the relative role of hillslope and network geometry in hydrologic response, Reidel, 1986.

Ogden, F. L. and Dawdy, D. R.: Peak discharge scaling in small Hortonian watershed, J. Hydrol. Eng., 8, 64-73, 2003.

Olivera, F. and Koka, S.: Hydrodynamic dispersive and advective processes in watershed responses, J. Hydrol. Eng., 9, 534-543, doi:10.1061/(ASCE)1084-0699(2004)9:6(534), 2004.

Olivera, F. and Maidment, D.: Geographic Information Systems (GIS)-based spatially distributed model for runoff routing, Water Resour. Res., 35, 1155-1164, 1999.

Rigon, R., Rodríguez-Iturbe, I., and Ijjasz-Vasquez, E.: Optimal channel networks: A framework for the study of river basin mor- 
phology, Water Resour. Res., 29, 1635-1646, 1993.

Rinaldo, A. and Rodríguez-Iturbe, I.: Geomorphological theory of the hydrological response, Hydrol. Process., 10, 803-829, 1996.

Rodríguez-Iturbe, I. and Rinaldo, A.: Fractal river basins: chance and self-organization, Cambridge University Press, New York, 1997.

Shreve, R.: Statistical law of stream numbers, J. Geol., 74, 17-37, 1966.

Troutman, B. M.: Scaling of flow distance in random self-similar channel networks, Fractals, 13, 265-282, 2005.

Troutman, B. M. and Karlinger, M. R.: Asymptotic Rayleigh Instantaneous unit-hydrograph, Stoch. Hydrol. Hydraul., 2, 73-78, 1988.
Troutman, B. M. and Over, T. M.: River flow mass exponents with fractal channel networks and rainfall, Adv. Water Resour., 24, 967-986, 2001.

Veitzer, S. and Gupta, V. K.: Random self-similar river networks and derivations of Horton-type relations exhibiting statistical simple scaling, Water Resour. Res., 36, 1033-1048, 2000.

Veitzer, S. and Gupta, V. K.: Statistical self-similarity of width function maxima with implications to floods, Ad. Water Resour., 24, 955-965, 2001.

Veitzer, S., Troutman, B., and Gupta, V. K.: Power-law tail probabilities of drainage areas in river basins, Phys. Rev. E, 68, 016123, doi:10.1103/PhysRevE.68.016123, 2003. 